\title{
Increasing the Specific Removal Rate for Ultra Short Pulsed Laser-Micromachining by Using Pulse Bursts
}

\author{
Torsten Kramer ${ }^{* 1}$, Yiming Zhang ${ }^{* 1}$, Stefan Remund ${ }^{* 1}$, Beat Jaeggi ${ }^{* 1}$, Andreas Michalowski ${ }^{* 2}$, \\ Lisa Grad*2 and Beat Neuenschwander*1 \\ ${ }^{* 1}$ Institute for Applied Laser, Photonics and Surface Technologies ALPS, \\ Bern University of Applied Sciences, Pestalozzistrasse 20, 3400 Burgdorf, Switzerland \\ E-mail: beat.neuenschwander@bfh.ch \\ *2 Laser Material Processing (CR/APJ2), Robert Bosch GmbH, \\ Renningen, 70465 Stuttgart, Germany \\ E-mail: Andreas.michalowsi@de.bosch.com
}

\begin{abstract}
The influence of the burst mode is investigated for steel, copper, silver and gold. It is shown that the gain in the removal rate, reported in literature, is generally caused by the lower fluence of the single pulses in the burst which is nearer the optimum value showing higher efficiency. Therefore, identical or even better results are obtained with single pulses of the same fluence but at higher repetition rate. However, for copper, silver and gold and a three pulse burst the second pulse hinders the ablation of the first pulse and it is supposed that it even re-deposits material. But compared to the first pulse the energy of the third pulse is much better converted to removed material leading to a real gain of $16 \%$ in efficiency for copper. An explanation of this typical behavior and its absence in the case of steel can be given by models of the ablation process dealing with a bulging and/or spalled layer and plasma as well.
\end{abstract}

DOI: 10.2961/jlmn.2017.02.0011

Keywords: burst mode, surface quality, laser micromachining, increased efficiency, ultra - short pulses, heat accumulation

\section{Introduction}

Ultra-short laser pulses have shown their applicability for high quality laser micromachining of metals, semiconductors and insulators in manifold applications. However, to effectively enter into the large field of industrial applications the demand of high throughput still represents one of the key factors. The burst mode was identified as a method increasing the throughput. As most ultra-short pulsed laser systems are arranged in a MOPA arrangement pulse bursts are basically generated as illustrated in fig. 1: The pulse picker reduces the repetition rate $f s$ of the seed oscillator to the desired repetition rate $f_{L}$ of the laser system. This picker is capable of letting pass not only single pulses but a sequence of $n$ pulses, which is then amplified in the following stages. The bursts are following each other with the repetition rate $f_{L}$, i.e. a time spacing of $\Delta t_{L}=1 / f_{L}$, whereas the time spacing between the pulses in the burst is given by $\Delta t_{B}=1 / f_{s}$. The highest possible number $n_{\max }$ of pulses in a burst and $\Delta t B$

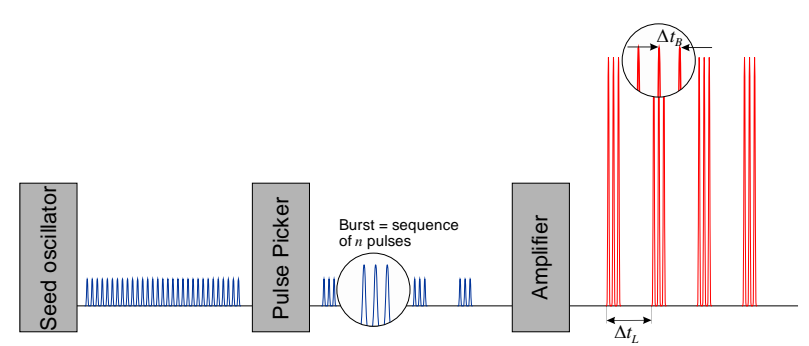

Fig. 1 Basic principle of burst generation. depend on the laser system. Some systems offer the possibility to suppress individual pulses in the burst sequence or even to adjust its energy.

Although bursts for ultra-short pulsed lasers are known for years, systematic studies for laser micromachining with bursts are only rarely published. In the case of glasses bursts are used e.g. for welding [1], writing of gratings or waveguides [2,3] or cutting [4-6]. For steel C75 and 12 ps pulses the influence of $\Delta t_{B}$ was investigated in steps of $20 \mathrm{~ns}$ for two pulse bursts [7]. It was shown, that for a two pulse burst containing the total energy of $22 \mu \mathrm{J}$ the ablated depth per layer is about $10 \%$ higher than twice the one obtained with single pulses at a pulse energy of $11 \mu \mathrm{J}$. But for a burst energy of $4 \mu \mathrm{J}$, corresponding to a single pulse energy of $2 \mu \mathrm{J}$ no increase was detected. In both cases rougher surfaces were obtained with bursts up to $\Delta t_{B} \approx 700 \mathrm{~ns}$. In [8] results from an adapted two temperature model simulation for a 5 pulse burst with $\Delta t_{B}=20 n s$ were compared with experimental results for copper by measuring the removed volume of machined dimples at $10 \mathrm{kHz}, 50 \mathrm{kHz}$ and $100 \mathrm{kHz}$ repetition rate. A good agreement between simulations and experiments as well as a significant higher ablated volume per pulse and time were observed for the 5 pulse burst. Heat accumulation and resulting stronger electron - phonon coupling are supposed to be responsible for this behavior. A dramatic increase of up to a factor of 14 in the specific removal rate compared to single pulses was reported for an 8 or 10 pulse burst when machining tungsten carbide and silicon [9] and even for stainless steel a 10 times higher specific removal rate was observed. 
But one has to have in mind that the removal rate also depends on the fluence and hence on the pulse energy itself [10-12]. In case of a Gaussian beam the specific removal rate as a function of the applied peak fluence $\phi_{0}$ reads:

$$
\frac{d V}{d E}=\frac{d V}{d t \cdot P_{a v}}=\frac{1}{2} \cdot \frac{\delta}{\phi_{0}} \cdot \ln ^{2}\left(\frac{\phi_{0}}{\phi_{t h}}\right)
$$

With $\delta$ the energy penetration depth, $\phi_{t h}$ the threshold fluence and $\phi_{0}=2 E_{p} / \pi w_{0}^{2}$. This function shows a peak value i.e. a maximum specific removal rate at an optimum fluence $\phi_{0, \text { opt }}$. The corresponding values are given by:

$$
\left.\frac{d V}{d E}\right|_{\max }=\frac{2}{e^{2}} \cdot \frac{\delta}{\phi_{t h}} ; \phi_{0, o p t}=e^{2} \cdot \phi_{t h}
$$

Therefore, just to compare removal rates can lead to strong misinterpretations as it can be illustrated with the data for stainless steel obtained in [9]. If the removal rate is shown in function of the laser repetition rate (at a constant average power) single pulses seem indeed to be much less efficient, as illustrated in fig. 2a. But if the specific removal rate is plotted as a function of the energy of the single pulses in the burst, as shown in fig. $2 \mathrm{~b}$, it becomes clear that this pulse energy represents the determining factor. Further, the solid curve in fig. $2 b$, representing the least square fit of the model function (1), suggests that the experiments were even

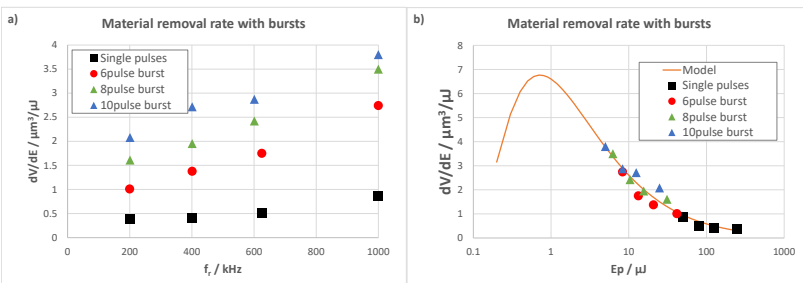

Fig. 2 Specific removal rates in stainless steel with $50 \mathrm{~W}$ average power deduced from [9], a) as a function of the repetition rate and $b$ ) as a function of the energy per single pulse in the burst. The solid line represents the least square fit of the model function (1) to the experimental data resulting in $\mathrm{w}_{0}=11 \mu \mathrm{m}$, $\delta=12 \mathrm{~nm}, \phi_{t h}=0.051 \mathrm{~J} / \mathrm{cm}^{2}$ and a maximum specific removal rate of $6.8 \mu \mathrm{m}^{3} / \mu \mathrm{J}$.

performed far away from the optimum point with maximum removal rate (2).

Thus, to have a fair comparison, the removal rate of a $\mathrm{n}$ - pulse burst at repetition rate $f_{r, B}$ should be compared with the one of single pulses at a repetition rate of $f_{r, s}=n \cdot f_{r, B}$. Taking this into account most of the improvements concerning the removal rate, reported for bursts, can be explained just by the lower energy per pulse in the burst which is nearer to its optimum value of $E_{p, \text { opt }}=\pi \cdot w_{0}^{2} \cdot \phi_{0, \text { opt }} / 2$ following (2).

To distinguish between the influence of the pulse energy of a single pulse in the burst and additional effects (e.g. heat accumulation) caused by the short time spacing $\Delta t_{B}$ in a burst, the specific removal rate was measured as a function of the peak fluence of a single pulse ranging from the threshold up to ten times its optimum value. Only if the maximum value of the specific removal rate obtained with a burst exceeds the one of single pulses, it has a real higher efficiency. Not answered with this study is the question of scalability i.e. if it is possible to scale up the average power by keeping the fluence near its optimum value and maintaining the removal rate and the machining quality. First experiments [13] have shown that copper and brass can be machined with more than $300 \mathrm{~W}$ of average power and a removal rate exceeding $40 \mathrm{~mm}^{3} / \mathrm{min}$ by maintaining a good surface quality. In contrast steel 1.4301 (AISI 304) shows a high heat accumulation [14] leading to a bumpy surface for an average power in the range of $100 \mathrm{~W}$ and a spot size of $\mathrm{w}_{0}=28 \mu \mathrm{m}$.

\section{Experimental Set-Up}

To deduce the specific removal rate squares with a side length of $s=1 \mathrm{~mm}, 1.6 \mathrm{~mm}$ or $2 \mathrm{~mm}$ were machined into a metallic target with a constant pitch (distance from pulse to pulse or line to line) of $p=8 \mu \mathrm{m}$ in $x$ and $y$ direction. This was realized by using a synchronized galvo - scanner [15] with an $\mathrm{f}=160 \mathrm{~mm} \mathrm{f}-$ theta objective resulting in a spot size of about $w_{0}=16 \mu \mathrm{m}$. A FUEGO $10 \mathrm{ps}-$ laser system offering a maximum average power above $40 \mathrm{~W}$ at the fundamental wavelength of $1064 \mathrm{~nm}$ was used as laser source. With a $\lambda / 4$ - plate the linear polarization was transformed to circular. The repetition rate can be varied between $200 \mathrm{kHz}$ and $8 \mathrm{MHz}$. Via the FlexBurst ${ }^{\mathrm{TM}}$ option a burst up to 8 pulses with a time spacing of $\Delta t_{B}=12 \mathrm{~ns}$ can be generated whereas the energy of each pulse in the burst can be adjusted individually.

With the laser repetition rate $f_{L}$, the pitch $p$, the number of machined slices $N_{S l}$ and the depth $d$ of the squares, measured with a white light interference microscope (smart WLI from gbs), the specific removal can then be calculated by:

$$
\frac{d V}{d E}=\frac{\Delta V}{E_{p}}=\frac{d \cdot p^{2}}{N_{S l}} \cdot \frac{f_{L}}{P_{a v}}=\frac{d \cdot p^{2}}{N_{S l}} \cdot \frac{1}{E_{p}}
$$

The number of slices was chosen in a way that the number of single pulses per area could be kept constant e.g. for a 6 pulse burst the number of slices had to be 6 times smaller compared to the one for single pulses. As 1, 2, 3, 4, 6 and 8 pulses per burst were investigated the number of slices for single pulses was 192, 96 or 48 . The metals investigated in this study were copper DHP (C12 200), steel 1.3401 (AISI 304), silver and gold. The repetition was basically set to $200 \mathrm{kHz}$ but to demonstrate the scalability repetition rates up to $1 \mathrm{MHz}$ and $1.6 \mathrm{MHz}$ were tested for copper and steel, respectively.

Beside the specific removal rate also the amount of the incoming energy which is converted to heat was measured for steel and copper with a calorimetric set-up following $[14,16]$.

\section{Experimental Results}

\subsection{General Results for Single Pulses}

To demonstrate the scalability of the ablation process, steel and copper were machined with a constant pitch and peak fluence up to a repetition rate of $1.6 \mathrm{MHz}$ and $2 \mathrm{MHz}$, respectively. No significant influence of the repetition rate onto the specific removal rate up into this regime was observed. As an example the results for steel are summarized in fig. 3. It has to be pointed out that the applied average power for the repetition rate of $1.6 \mathrm{MHz}$ was 8 times higher than that for $200 \mathrm{kHz}$ for the same value of peak fluence. Steel shows a special behavior concerning high fluences. If 
its value exceeding about 1.5 times the optimum value cavity formation starts and degrades the machining quality. This cavity formation becomes stronger with increasing fluence and will finally cover the whole surface as e.g. shown in [17]. This cavity formation additionally leads to a reduced removal rate and finally the specific removal rate does not follow the model function (1) for higher fluences. Hence, steel should not be machined with fluences exceeding 1.5 times its optimum value. In contrast for all other investigated metals the deduced specific removal rate exactly follows the model function (1) when the fluence is raised.

The obtained results for the optimum fluence and the corresponding maximum removal rate for the investigated metals steel, copper, silver and gold are summarized in tab. 1. Sake of completeness also the values for brass and nickel are added.

The highest maximum removal rates of about $5 \mu \mathrm{m}^{3} / \mu \mathrm{J}$ $\left(0.3 \mathrm{~mm}^{3} / \mathrm{min} / \mathrm{W}\right)$ are obtained for the noble metals gold and silver whereas for copper and steel it only amounts about $2.25 \mathrm{\mu m}^{3} / \mu \mathrm{J}$.

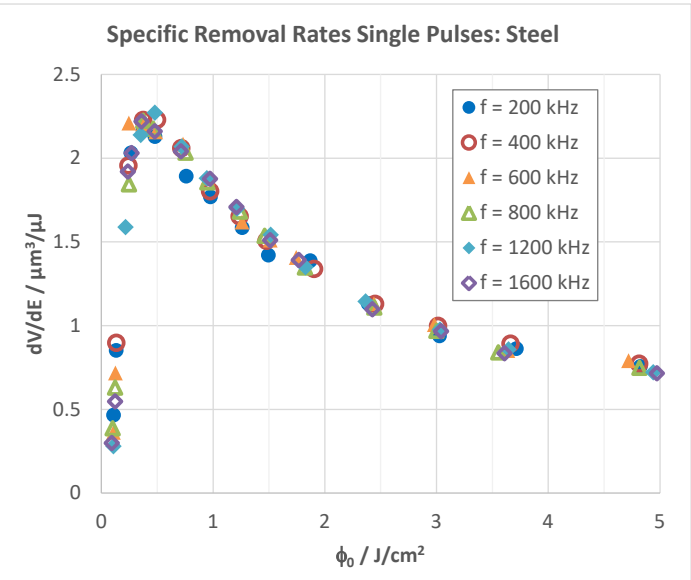

Fig. 3 Specific removal rates in stainless steel as a function of the peak fluence obtained by machining squares with 192 slices and repetition rates between $200 \mathrm{kHz}$ and $1.6 \mathrm{MHz}$.

Table 1 Optimum peak fluence and maximum specific removal rates for different metals

\begin{tabular}{ccc}
\hline Material & $\phi_{0, \mathrm{opt}} / \mathrm{J} / \mathrm{cm}^{2}$ & $d V / d E_{\max } / \mu \mathrm{m}^{3} / \mu \mathrm{J}$ \\
\hline AISI 304 & 0.5 & 2.25 \\
C12 200 & 2.65 & 2.22 \\
Ag & 2.68 & 5.02 \\
Au & 2.41 & 5.04 \\
CuZn39Pb2 & 1.9 & 3.87 \\
Ni & 0.65 & 1.93 \\
\hline
\end{tabular}

\subsection{Bursts on Steel}

In a first experiment the specific removal rate of a two pulse burst was deduced for time spacing $\Delta t_{B}$ of $12 \mathrm{~ns}, 24 \mathrm{~ns}$, $36 \mathrm{~ns}$ and $60 \mathrm{~ns}$. The results for the first 3 spacings are shown in fig. 4. Compared to single pulses the maximum specific removal drops by about $20 \%$ from $2.25 \mu \mathrm{m}^{3} / \mu \mathrm{J}$ to
$1.85 \mu \mathrm{m}^{3} / \mu \mathrm{J}$ for $\Delta t_{B}=12 n s$. For $24 \mathrm{~ns}$ the drop is reduced to about $3 \%$ and it completely vanishes for 36 ns and also 60 ns (not shown here). Hence one can conclude that the specific removal rate for a burst with $\Delta t_{B}>24 n s$ exactly follows the curve of single pulses. Similar results are obtained for a 3 pulse burst as shown in fig. 5 . The drop in the maximum value of the specific removal rate amounts 25\%, $15 \%$ and $10 \%$ for a time spacing of 12 ns. 24 ns and 36 ns. In both cases the value of the optimum fluence is shifted to higher fluences for longer time spacing.

The specific removal rates for a 2, 3, 4, 6 and 8 pulse

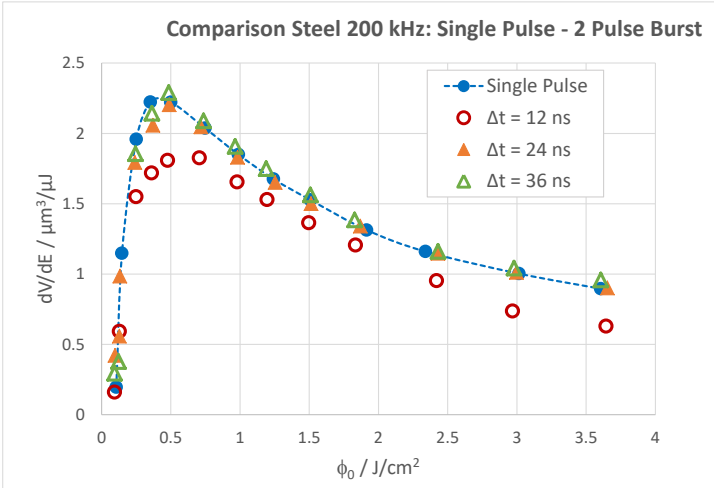

Fig. 4 Specific removal rate of a 2 pulse burst for stainless steel as a function of the time spacing between the burst pulses.

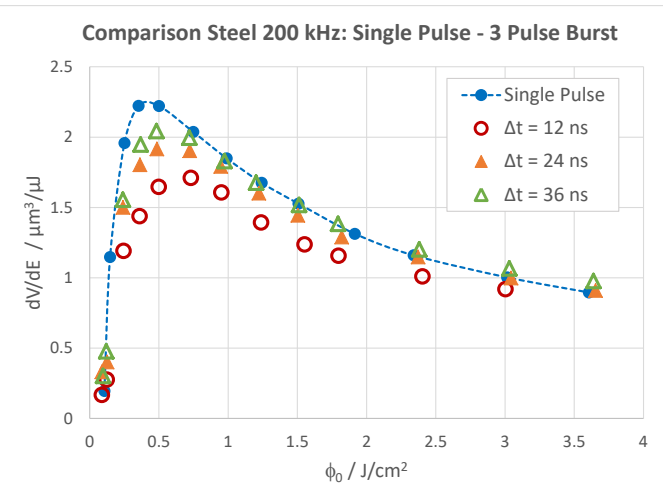

Fig. 5 Specific removal rate of a 3 pulse burst for stainless steel as a function of the time spacing between the burst pulses.

burst are shown in fig. 6 . The maximum specific removal

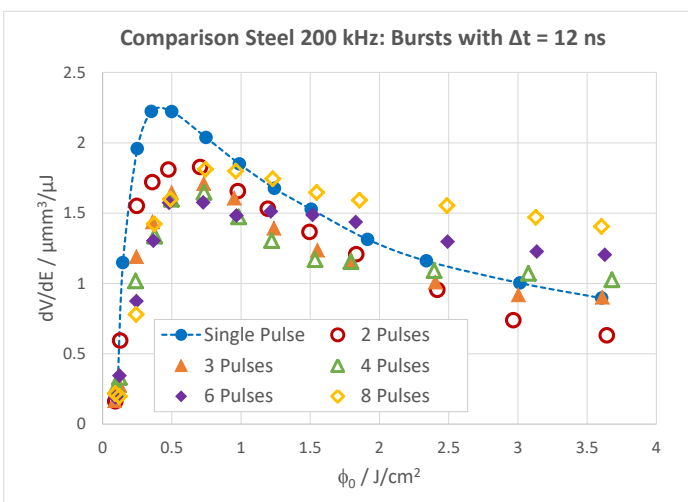

Fig. 6 Specific removal rate in stainless steel of a 2, 3, 4, 6 and 8 pulse burst. 
rate first drops up to 6 pulses per burst and slightly increases for 8 pulses per burst and the value for the optimum fluence is shifted to higher values. The highest value of the specific removal rate is obtained for single pulses. Taking also the results for the 2- and 3- pulse burst with different time spacing into account there exist no situation where a higher maximum specific removal rate can be obtained than for single pulses. Only for a peak fluence exceeding $1.5 \mathrm{~J} / \mathrm{cm}^{2}$ there could be a benefit in efficiency if a burst with 6 or 8 pulses is applied.

\subsection{Bursts on Copper}

The results for a 2 pulse burst with different spacing between 12 ns and 60 ns are summarized in fig. 7. In contrast to steel a dramatic drop in the specific removal rate of $60 \%$ for a spacing of $12 \mathrm{~ns}, 58 \%$ for $24 \mathrm{~ns}, 53 \%$ for $36 \mathrm{~ns}$ and $40 \%$ for 60 ns can be observed. A drop higher than $50 \%$ means that the second pulse redeposits already ablated material or hinders the removal process of the first pulse does itself not remove any material.

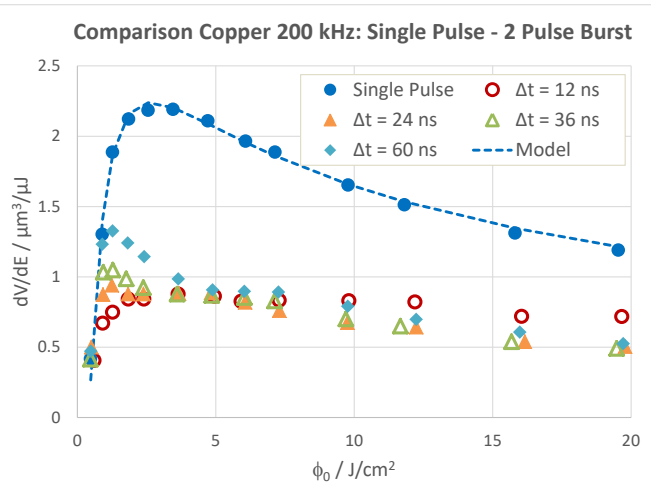

Fig. 7 Specific removal rate of a 2 pulse burst for copper as a function of the time spacing between the burst pulses.

A completely surprising result is obtained for 3 pulse bursts. As illustrated in fig. 8 the maximum specific removal rate increases for a time spacing of 12 ns by about $16 \%$ compared to single pulses. Considering the drop of $60 \%$ for a two pulse burst the $3^{\text {rd }}$ pulse seems to strongly boost the removal process. For a time spacing of $24 n s$ and 36ns the maximum specific removal rate is lower compared to single pulses. But also for these situations the $3^{\text {rd }}$ pulse seems to be much more efficient having the 2 pulse burst behavior in

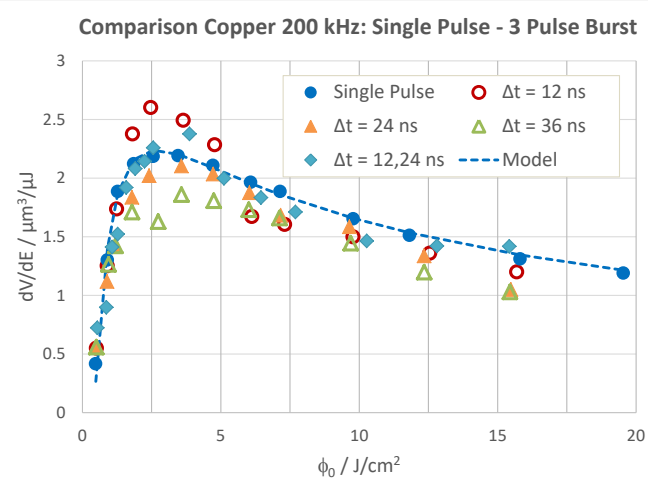

Fig. 8 Specific removal rate of a 3 pulse burst for copper as a function of the time spacing between the burst pulses. mind. A special burst with 12 ns between the $1^{\text {st }}$ and the $2^{\text {nd }}$ pulse and $24 \mathrm{~ns}$ between the $2^{\text {nd }}$ and the $3^{\text {rd }}$ pulse did not lead to a higher gain in the removal rate.

For a further investigation the energy of the $2^{\text {nd }}$ pulse in the burst with $\Delta t_{B}=12 n s$ was adjusted to $0,25 \%, 50 \%$, $75 \%$ and $100 \%$ of the $1^{\text {st }}$ and $3^{\text {rd }}$ pulse energy. The peak fluence is then referred to the $1^{\text {st }}$ and the $3^{\text {rd }}$ pulse in the burst but to deduce the specific removal rate the reduced energy for the $2^{\text {nd }}$ pulse has to be considered. These experiments were performed at a repetition rate of $1 \mathrm{MHz}$ and are sum-

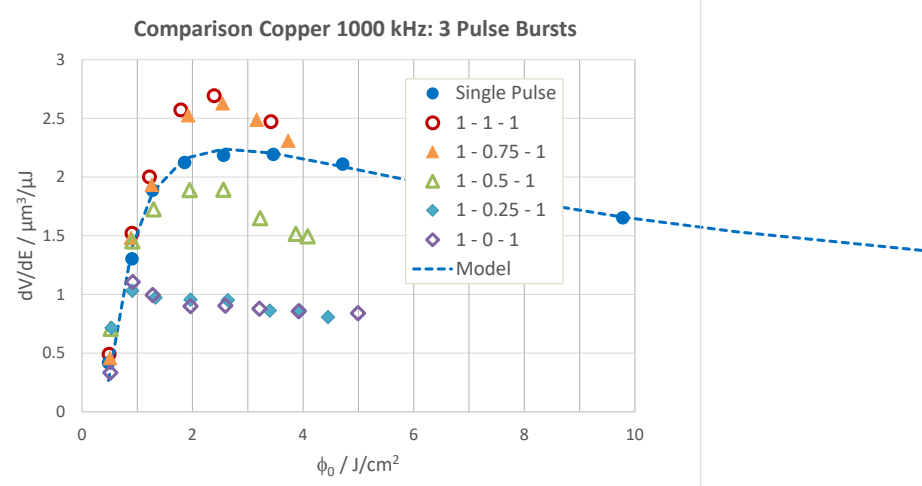

Fig. 9 Specific removal rate of a 3 pulse burst for copper as a function of the energy of the $2^{\text {nd }}$ pulse in the burst.

marized in fig. 9. The specific removal rate does not significantly change when the energy of the $2^{\text {nd }}$ pulse is reduced to $75 \%$. A strong drop starts when it is further reduced to $50 \%$ and $25 \%$. For the latter equal removal rates as for a 2 pulse burst with a spacing of 24 ns are obtained. I.e. varying the energy of the $2^{\text {nd }}$ pulse between $25 \%$ and $75 \%$ leads to strong variations in the maximum specific removal rate.

The results for bursts with 2, 3, 4, 6 and 8 pulses with a

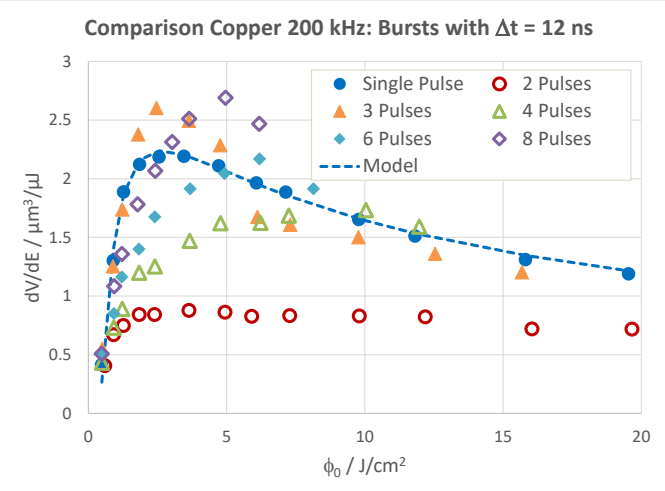

Fig. 10 Specific removal rate in copper of a 2, 3, 4, 6 and 8 pulse burst.

spacing of 12 ns are summarized in fig. 10. The 4 and 6 pulse burst do not exceed the maximum specific removal rate for single pulses but for 8 pulses in the burst the value of a 3 pulse burst is almost obtained but at a higher peak fluence. However, for the 8 pulse burst the machined squares show a higher roughness and are blackened as shown in [17].

\subsection{Bursts on Silver}

Compared to copper and steel silver shows a maximum specific removal rate which is about two times higher. As the threshold fluence of $\phi_{t h}=0.36 \mathrm{~J} / \mathrm{cm}^{2}$ is in the same order as 
for copper this increase is mainly caused by the much higher energy penetration depth of $\delta=67 \mathrm{~nm}$. Similar to copper a

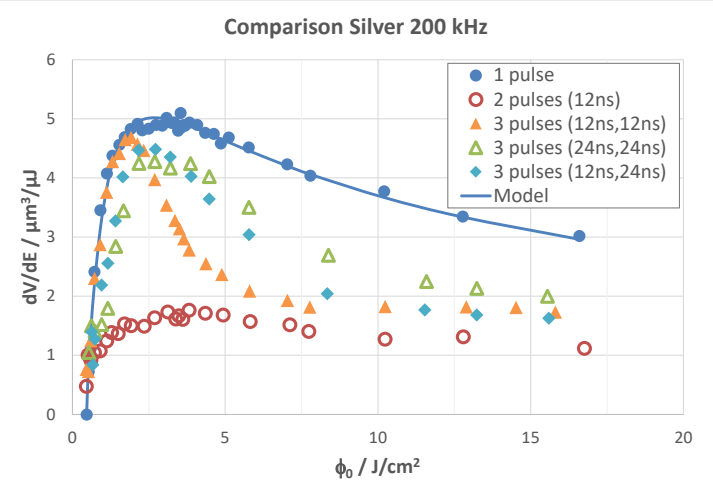

Fig. 11 Specific removal rate for silver of single pulses, 2 pulse burst with 12 ns spacing and 3 pulse bursts with different time spacing.

dramatic drop of the specific removal rate for a 2 pulse burst with a spacing of 12 ns is observed. With 65\% this drop is even higher than the one for copper. Again the 3 pulse burst with a spacing of 12 ns between the pulses is much more efficient but it does not exceed the values obtained for single pulses. Also different time spacing (24 ns-24 ns and 12 ns - 24 ns) do not lead to higher removal rates as illustrated in fig. 11 .

\subsection{Bursts on Gold}

Similar results as for silver are also obtained for gold as shown in fig. 12. As the threshold fluence of $\phi_{t h}=0.33 \mathrm{~J} / \mathrm{cm}^{2}$ and an energy penetration depth of $\delta=60.8 \mathrm{~nm}$ are very similar to silver also the maximum specific removal rate does hardly differ as shown in tab. 1 . The drop in the removal rate

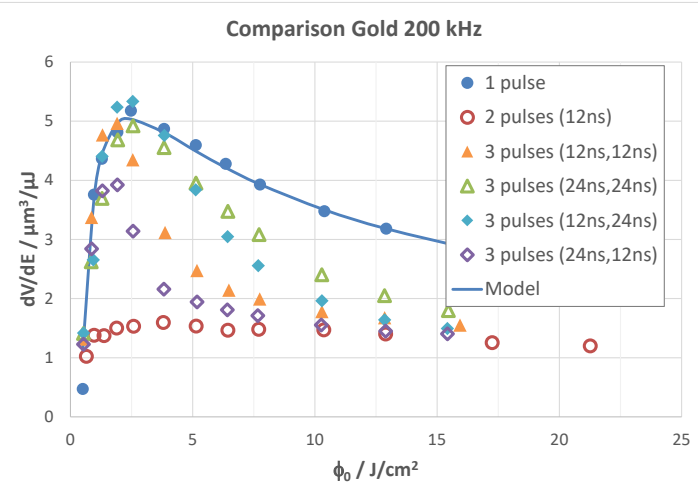

Fig. 12 Specific removal rate for gold of single pulses, 2 pulse burst with $12 \mathrm{~ns}$ spacing and 3 pulse bursts with different time spacing.

for the 2 pulse burst with a spacing of 12 ns amounts about $69 \%$ which is the highest value observed for the four investigated materials. The 3 pulse burst was investigated with all combinations of $12 \mathrm{~ns}$ and $24 \mathrm{~ns}$ time spacing. A spacing of 12 ns or 24 ns between all pulses lead to a maximum specific removal rate which reaches almost the value for single pulses. But for 12 ns a strong drop in the removal rate is observed for peak fluences exceeding $2 \mathrm{~J} / \mathrm{cm}^{2}$. For $24 \mathrm{~ns}$ this drop starts later between 2.5 and $3.5 \mathrm{~J} / \mathrm{cm}^{2}$ and is less pronounced. If the first spacing is short (12ns) and the second long (24ns) the maximum value of the specific removal rate seems to exceed the one of single pulses but as this gain may be within the measurement error no clear statement can be made.

\section{Discussion}

\subsection{Single Pulses and Two Pulse Burst}

For all four investigated metals a drop in the specific removal rate for a 2 pulse burst has been observed. For steel and copper it has been shown that this drop decreases with increasing time spacing between the two pulses. This behavior is much more pronounced for copper where the drop for a spacing of 60 ns still amounts $40 \%$. For steel the values of single pulses are obtained already for a spacing of only 24 ns. Similar behavior as for copper is expected for silver and gold. The significantly reduced drop for steel may be caused by the much smaller volume or mass ablated per pulse at the optimum point. Using (1) leads to:

$$
d V_{\max }=\delta \cdot \pi \cdot w_{0}^{2}
$$

The ablated volume per pulse then amounts $\left(\mathrm{w}_{0}=15.5 \mu \mathrm{m}\right) 4.1 \mu \mathrm{m}^{3}$ for steel, $22.4 \mu \mathrm{m}^{3}$ for copper, $50.7 \mu^{3}$ for silver and $45.9 \mu^{3}$ for gold. The corresponding masses are $0.03 \mathrm{ng}, 0.2 \mathrm{ng}, 0.53 \mathrm{ng}$ and $0.89 \mathrm{ng}$. Thus, for steel the removed material or mass per pulse is more than 5 times smaller. Huge differences are also observed in the

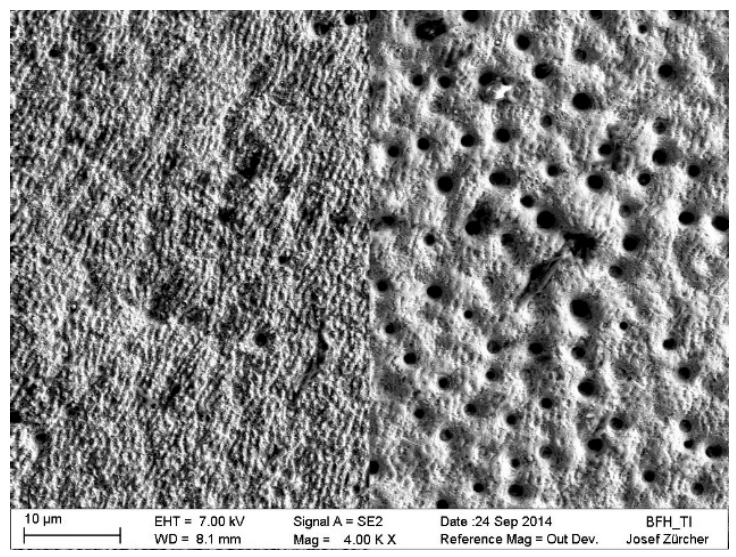

Fig. 13 SEM micrograph of the steel surface machined with optimum fluence for single pulses at $200 \mathrm{kHz}$. Left: single pulses. Right: 2 pulse burst (12ns)

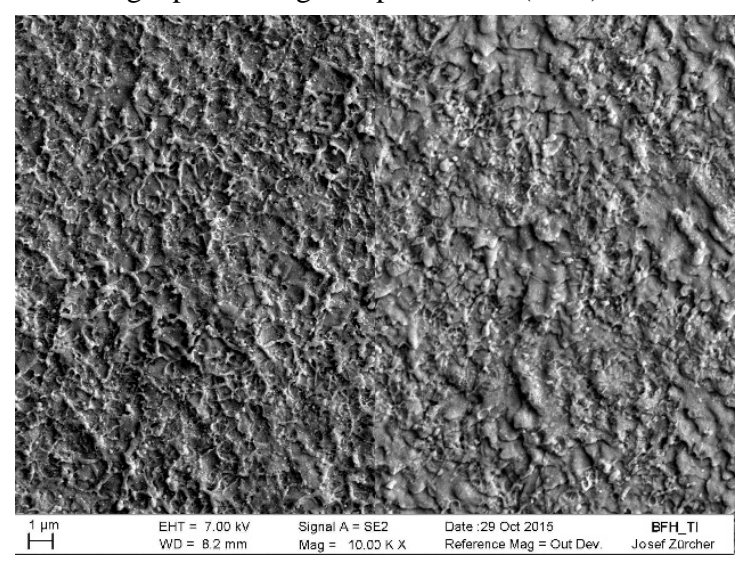

Fig. 14 SEM micrograph of the copper surface machined with optimum fluence for single pulses at $200 \mathrm{kHz}$. Left: single pulses. Right: 2 pulse burst (12ns)

micrographs. Fig. 13 shows SEM micrographs of the steel surface machined with single pulses (left) and a two pulse 
burst (right) with a time spacing of 12 ns at the optimum fluence. The single pulse surface shows some small structures and first small cavities can be observed. For the two pulse burst the number of cavities is strongly increased and the structure of the surface equals the one of single pulses. For copper the surface machined with single pulses at the optimum fluence is covered by small "melting splashes" as shown left in fig. 14. For the two pulse burst, shown on the right side, the surface seems to be covered by small melted plates with dimensions of a few $\mu \mathrm{m}$. Similar results are also observed for silver and gold.

This behavior can be linked to the ablation process. In [18] experiments with $200 \mathrm{fs}$ pulses on steel at a high fluence of $20 \mathrm{~J} / \mathrm{cm}^{2}$ have shown that the material removal occurs in two steps. The first ablation phase takes place immediately after the irradiation. This ablation phase is expected to appear due to plasma formation by direct ionization, sublimation, photoelectric and/or thermionic electron emission. The second ablation phase with a strong increase of the plasma luminescence intensity and the formation of a particle wave is delayed by $30-50$ ns with respect to the incident laser pulse. This delay was identified to be a clear indication of involvement of thermal processes like boiling and vaporization. Following [19] a thin layer of thickness d with material in an inhomogeneous phase is formed and starts to bulge after a time $t_{0}=d / c_{0}$ with $c_{0}$ the speed of sound in the material and $\mathrm{d}$ in the order of the ablation depth. The bulging of this layer continues until it finally leaves the target surface. The formation and bulging of this layer was found for all investigated material ( $\mathrm{Si}(111), \mathrm{GaAs}, \mathrm{Ti}, \mathrm{Au}$ and $\mathrm{Al}$ ) when they were irradiated with a $100 \mathrm{fs}$ pulse at $620 \mathrm{~nm}$. In contrast to the experiments in [18] the applied fluence was only moderate e.g. $0.43 \mathrm{~J} / \mathrm{cm}^{2}$ for Si. In [20] the two temperature model, describing the absorption of the optical energy and its transfer to the lattice, is combined with large scale molecular dynamic simulations to calculate the lattice reaction in case of aluminum. This calculations are done for single pulses of a pulse duration of $100 \mathrm{fs}$, a wavelength of $800 \mathrm{~nm}$ and different absorbed fluences from the threshold up to a multiple of it. Also in this work it was found that at least in the wings of the Gaussian beam a layer is formed wich will be spalled from the surface whereas in the center of the beam droplets are ejected. Assuming a surface reflectivity of $91 \%$ leads to applied fluences, used for the simulations, which are in the

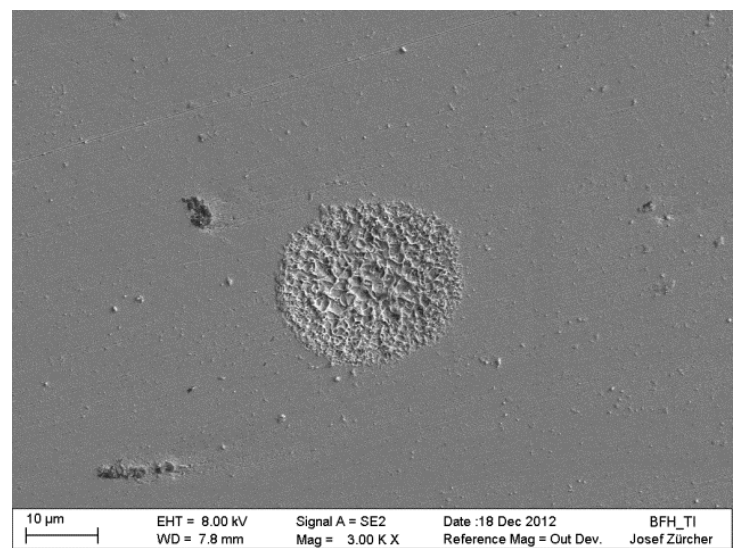

Fig. 15 SEM micrograph of the a crater machined in copper with 8 pulses at $50 \mathrm{~Hz}$. same order than the threshold fluence for single pulses measured for aluminum with $10 \mathrm{ps}$ pulses at $1064 \mathrm{~nm}$ in former experiments.

Based on these results the following assumption is made: The formation and bulging of the layer described in $[19,20]$ is assigned to the first ablation phase reported in [18] whereas for the second phase at least parts of this layer vaporize, cause the plasma luminescence and form the particle wave as well. The unvaporized part of this layer forming droplets [20] may partially fall back onto the surface and form the small melting splashes as shown for copper in the left part of fig. 14. Similar melting splashes were also observed for silver and gold as well as for craters machined in copper at a repetition rate of $50 \mathrm{~Hz}$ [21] as shown in fig. 15. The low repetition rate indicates that these splashes were not formed by heat accumulation and were generated by the ablation process itself.

If the optimum fluence going with the maximum specific removal rate for single pulses is applied the ablation depth is in the range of the energy penetration depth and together with $\mathrm{c}_{0}$ the time until the bulging of the layer starts can be estimated. It amounts about 1 ps for steel, 6 ps for copper and 18 ps for silver and gold. All values are far below the minimum time spacing of 12 ns between the burst pulses. As the energy penetration depth for steel is below $10 \mathrm{~nm}$ the bulging layer would be very thin or is even destroyed when the second pulse of a burst will impinge on it whereas for the other three metals the layer could still be of notable thickness and be expanding. The second pulse will therefore be partially be absorbed by this expanding layer for copper, silver and gold. The other part of the pulse is assumed to be scattered, reflected or also absorbed by the vaporized part of the ablated material. This could finally lead to a complete shielding of the second pulse and a redirection of a part of the bulging layer and thus to a re - deposition of liquid material on the surface. This is a possible explanation of the drop in the specific removal rate below $50 \%$ of the value obtained for single pulses and the appearance of the surface as it was observed on copper, silver and gold. The not redirected part of the expanding layer and the ejecting droplets should then have higher energy and can therefore expand even faster or it's boiling and vaporization starts earlier.

\subsection{Three Pulse Burst}

Also the behavior of the third pulse can partially be explained by the model. For higher fluences exceeding the optimum value for single pulses the specific removal rate of copper roughly follows the one for single pulses whereas it strongly drops towards the values of a two pulse burst for silver and gold. Compared to copper, these two materials show a doubled energy penetration depth going with a thicker expanding layer. Therefore it may still be present when the $3^{\text {rd }}$ pulse arrives and can partially absorb, scatter or reflect its energy.

In the case of copper, silver and gold and for moderate fluences around or below the optimum value for single pulses the differences in the specific removal rate (for a three pulse burst) to single pulses is much smaller than to the two pulse burst. This means that the energy of the third pulse is converted into removed material with much higher efficiency. This could be explained by the assumption that the second 
pulse lead to a faster evaporation and expansion of the bulging layer and that the redirected part could be melted. In ideal case the third pulse will not be attenuated by particle or plasma shielding which is, following [23], expected to be small at our applied fluences. Further the pulse impinges on a partially molten surface. Compared to single pulses the surface is rougher and covered by bigger melting splashes for a three pulse burst as illustrated in fig. 16 for copper and silver. In [23] it is reported that for a wavelength of $10.6 \mu \mathrm{m}$ the absorbance of copper in the liquid phase is about 3 times higher than in the solid phase. If this would also hold for $1064 \mathrm{~nm}$ this could be responsible for the higher efficiency in the conversion of the incoming energy to ablated material for the third pulse in the burst and could finally also explain the $16 \%$ higher maximum specific removal rate obtained compared to single pulses. In [24] the refractive and absorption index of silver and gold was measured in solid and liquid phase. From the obtained values the absorbance can be calculated. It equals about $1 \%$ in the solid and $9 \%$ in the liquid phase for gold. Therefore an even higher removal rate for the three pulse burst could be expected. But with no combination of three burst pulses a significantly higher maximum specific removal rate compared to single pulses was observed. A possible explanation could be found in the higher drop for a two pulse burst of $69 \%$ compared to $60 \%$ which could lead to still higher absorption, reflection and

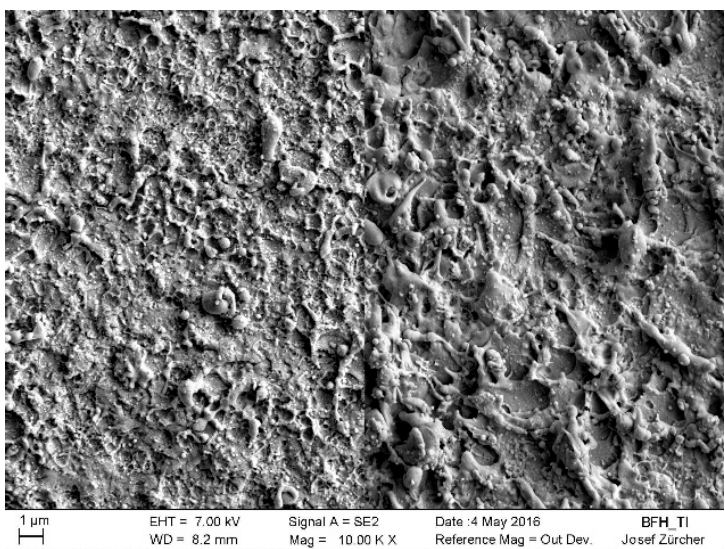

Fig. 16 SEM micrograph of the copper (left) and silver (right) surface machined with a 3 pulse burst at optimum fluence for single pulses at $200 \mathrm{kHz}$.

scattering of the third pulse of the burst in the residual layer and droplets. The higher specific removal rate for a burst with 12 ns and 24 ns spacing compared to $12 n s$ and 12 ns or 24 ns and 12 ns confirms this assumption.

But for silver, showing almost the same behavior as gold the absorbance in the solid and liquid phase would be $2.2 \%$ and $2.5 \%$. If the higher efficiency of the third pulse would only be caused by the higher absorbance silver should show a completely different behavior.

Further the residual heat i.e. the part of the incoming energy converted to heat in the sample was measured according to $[14,16]$. If this energy would mainly be defined by the optically absorbed part, a lower heat for a two pulse burst (due to the blocked second pulse) and a much higher one for the three pulse burst (due to lower reflectivity) would be expected. For copper the results for a 1, 2, and 3 pulse burst and for a 3 pulse burst with varying energy of the second pulse are summarized in fig. 17. It can clearly be seen that the residual heat amounts about $25 \%$ of the incoming energy for all investigated number of pulses per burst. Also for steel it was almost independent of the number of pulses for a 1, 2 and 3 pulse burst. For the three pulse burst with increasing energy of the second pulse the residual heat seems to increase as well. But the measurement error don't allow a clear statement. As the applied method does not directly measure the optical absorption but the amount of the incoming energy converted to heat also additional effects as e.g. the redirected part of the expanding layer for the second pulse and any similar effect for the third pulse contribute to measured residual heat. This could finally explain the measured values but a higher precision in the experimental method is needed for clearer statements.

Taking this and the situation with the absorbance of copper, gold and silver in solid and liquid phase into account the situation for the third pulse rests unclear and has to be further investigated. If the time spacing would be variable and not limited to multiples of 12 ns a deeper understanding could help to design optimum combinations of the pulses in a three pulse burst resulting higher specific removal rates.

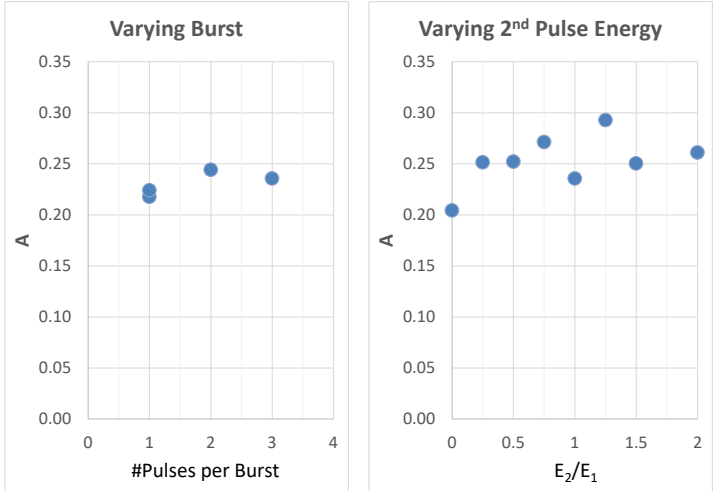

Fig. 17 Amount of the incoming energy converted to residual heat for a 1, 2 or 3 pulse burst (left) and for a 3 pulse burst with varying energy of the second pulse in the burst (right).

\section{Summary}

The specific removal rate for steel, copper, silver and gold for single pulses and pulse bursts as a function of the peak fluence of the single pulses (per burst) was investigated. It was found that the often reported benefit of the burst mode concerning the removal rate was mainly caused by the peak fluence of the single pulses in the burst which was nearer the optimum value needed for the maximum specific removal rate. Moreover, in general bursts lead to lower specific removal rates and only for copper and a three pulse burst a real benefit of this mode could be observed. For a two pulse burst always a significantly lower removal rate for copper, silver and gold was observed. Whereas for a three pulse burst the specific removal rate increases again and even exceeded in case of copper the one of single pulses by $16 \%$. Based on the literature a model of the ablation process was presented explaining the behavior of a two pulse burst and partially also for the three pulse burst. However, future investigations will be needed to clearly clarify the influence of the third pulse in the burst and the time spacing between the burst pulses.

But it has to be mentioned here that burst will have a benefit regarding the removal rate in the case that the used 
laser system or beam guiding system limits the repetition rate. In this case it can be more efficient to work with a burst instead of single pulses at higher energy.

\section{Acknowledgments}

This work was supported by the European Union via the project APPOLO (GA 609355).

The authors thank Josef Zuercher for the help with the SEM micrographs and Urs Hunziker for his work in the mechanical workshop.

\section{References}

[1] F. Zimmermann, S. Richter, S. Döring, A. Tünnermann, S. Nolte, Appl. Opt., 52, (2013)

[2] H. Zhang, S.M. Eaton, P.R. Herman, Opt. Lett., 32, (2007), 2559

[3] K. Itoh, JLMN J. of Laser Micro/Nanoengineering, 3, (2014), 187

[4] M. Kumkar, L. Bauer, S. Russ, M. Wendel, J. Kleiner, D. Grossmann, K. Bergner, S. Nolte, Proc. of SPIE, 8972, (2014)

[5] F. Hendricks, V.V. Matylitsky, Proc. of SPIE 9740, (2016)

[6] K. Mishchik, B. Chasagne, C. Javaux-Léger, C, Hoenninger, E, Mottay, R, Kling, J. Lopez, Proc. of SPIE 9740, (2016)

[7] C.A. Hartmann, T. Fehr, M. Braydic, A. Gillner, JLMN J. of Laser Micro/Nanoengineering, 2, (2007), 44

[8] W. Hu, Y.C. Shin, G. King, Appl. Phys. A, 98, (2009), 407

[9] R. Knappe, H. Haloui, A. Seifert, A. Weis, A. Nebel, Proc. of SPIE, 7585, (2010)

[10]G. Raciukaitis, M. Brikas, P. Gecys, B. Voisiat, M. Gedvilas, JLMN Journal of Laser Micro/Nanoengineering, 4, (2009), 186
[11]B. Neuenschwander, G. Bucher, Ch. Nussbaum, B. Joss, M. Muralt, U. Hunziker, P. Schuetz, Proc. of SPIE, 7584, (2010)

[12]B. Neuenschwander, B. Jaeggi, M. Schmid, Phys. Proceedia, 41, (2013), 794

[13]B. Jaeggi, M. Zimmermann, G. Hennig, R. Streubel, B. Goekce, S. Barciskowski, B. Neuenschwander, Proceedings of SLPC 2016, to be published in JLNM Journal of Laser Micro/Nanoengineering

[14]F. Bauer, A. Michalowski, Th. Kiedrowski, S. Nolte, Opt. Expr. 23, (2015), 1035

[15]B. Jaeggi, B. Neuenschwander, T. Meier, M. Zimmermann, G. Hennig, Physics Procedia, 41, (2013), 319 - 326

[16]F. Bauer, A. Michalowski, S. Nolte, JLMN-Journal of Laser Micro/Nanoengineering Vol. 10, (2015), 325

[17]B. Neuenschwander, T. Kramer, B. Lauer, B. Jaeggi, Proc. of SPIE 9350, (2015)

[18] S. Nolte, F. Schrempel, F. Dausinger: "Ultra Short Pulse Laser Technology” Springer Series in Opt. Sci. 195, (2016), 200

[19]D. von der Linde, K. Sokolowski-Tinten, Appl. Surf. Sci., (2000), 154

[20] Ch. Wu, L. Zhigilei, Appl. Phys. A, 114, (2014), 11

[21]B. Neuenschwander, B. Jaeggi, M. Schmid, A. Dommann, A. Neels, T. Bandi, G. Hennig, Proc. of SPIE 8607, (2013)

[22] J. Koenig, S. Nolte, A. Tuennermann, Opt. Express, 13, 26, (2005), 10597

[23] M. Brückner, J. H. Schäfer, J. Uhlenbusch, J. Appl. Phys. 66(3), (1989), 1326

[24]M. Schmid, S. Zehnder, P. Schwaller, B. Neuenschwander, J. Zürcher, U. Hunziker, Proc. of SPIE 8607, (2013)

(Received: May 22, 2016, Accepted: August 8, 2017) 